\title{
Microstructure and Texture Evolution of Pure Al Subjected to Torsion Deformation
}

\author{
Jie Liu ${ }^{1, a}$, Han Chen ${ }^{2, b *}$ \\ ${ }^{1}$ School of Aeronautics, Northwestern Polytechnical University, Youyixi Road, Xi'an, 710072, China \\ ${ }^{2}$ State Key Laboratory of Metal Matrix Composites, Shanghai Jiao Tong University, 800Dongchuan \\ Road, Shanghai, 200240, PR China \\ a15929958175@163.com, bchenhan590@mail.nwpu.edu.cn \\ *Corresponding Author: Han Chen
}

Keywords: Pure aluminum, torsion deformation, microstructure evolution, texture development, refinement mechanisms.

\begin{abstract}
Systematic experimental studies on microstructure evolution and texture development of pure Al during torsion at room temperature are carried out by electron back scattering diffraction (EBSD) and transmission electron microscopy (TEM). The microstructure evolution states the grains of deformed pure Al are refined evidently. The refinement mechanisms of pure Al are attributed to dislocation slip and recrystallization. The texture evolution of pure Al indicates that the position of $\{001\}$ fiber changes slightly, but its intensity first increases and then decreases with the increase of deformation during torsion. Finally, the processes of grain refinement of pure $\mathrm{Al}$ during torsion are discussed based on TEM observations.
\end{abstract}

\section{Introduction}

The torsion deformation is often used to improve the mechanical properties of metal materials by combining other deformation modes [1-2], for the torsion deformation can provide a higher plastic strain accumulation than tension and compression before fracture [3]. Therefore, the torsion deformation has been paid more attention to in recent years, and the deformation behaviors of some metal materials under torsion are studied by the present authors [4-5]. It is found that these researches concentrate on microstructure evolution and mechanical properties during torsion, which can not provide a complete description on microstructure sufficiently. And the detailed analysis concerning crystallographic orientations should be provided in order to comprehend microstructure characteristic better for polycrystalline materials [6]. It is well known that the crystallographic texturing resulting from the reorientation of the crystal lattices of grains can lead to anisotropic plastic response on polycrystalline metals. And as a result, it is of great importance to investigate texture evolution and its effect on the mechanical behavior during deformation [7].

Based on above studies, this paper aims at presenting the experimental results on microstructure and texture evolution of pure aluminum during torsion. And the effects of dislocation movement caused by torsion on microstructure evolution are discussed. The special studies of microstructure and texture evolution during torsion can be used in understanding and predicting anisotropic mechanical behavior, which will be beneficial to design for better control and energy efficiency improvement during forming processes.

\section{Experiments and Methods}

The torsion tests are performed on $\mathrm{XC}$ wire torsion test machine using annealed pure aluminum (1070). The gauge length of specimen is $\Phi 6 \mathrm{~mm} \times 60 \mathrm{~mm}$. In order to introduce fairly homogeneous and equiaxed microstructures, the pure $\mathrm{Al}$ is annealed at $553 \mathrm{~K}$ for $2 \mathrm{~h}$, then cooled down in furnace. The average maximum torsional turns are about 9.2, and the samples after twisting 3, 6 and 9 turns are used for microstructure and texture investigation. 
The samples for EBSD analysis are taken from longitudinal section of deformed billets. The EBSD samples are first mechanically polished and then electropolished. The pure $\mathrm{Al}$ is electropolished in a solution of $100 \mathrm{ml}$ perchloric acid and $900 \mathrm{ml}$ ethanol at $298 \mathrm{~K}$ using a DC power supply with $28 \mathrm{~V}$ in 40 seconds. The coordinate system is defined as: X0, Y0 (parallel to rods axis) and $\mathrm{Z0}$ (perpendicular to longitudinal section) refer to rolling direction (RD), transversal direction (TD) and nominal direction (ND) respectively. Finally, TEM specimens are punched from the longitudinal section of billets, and then polished mechanically. A solution of $330 \mathrm{ml}$ nitric acid and $670 \mathrm{ml}$ methanol is used to polish the deformed pure Al specimens by a twin-jet polishing unit at an applied potential of $40 \mathrm{~V}$ and a temperature of $233 \mathrm{~K}$.

\section{Results and Discussion}

\subsection{Microstructure Evolution}
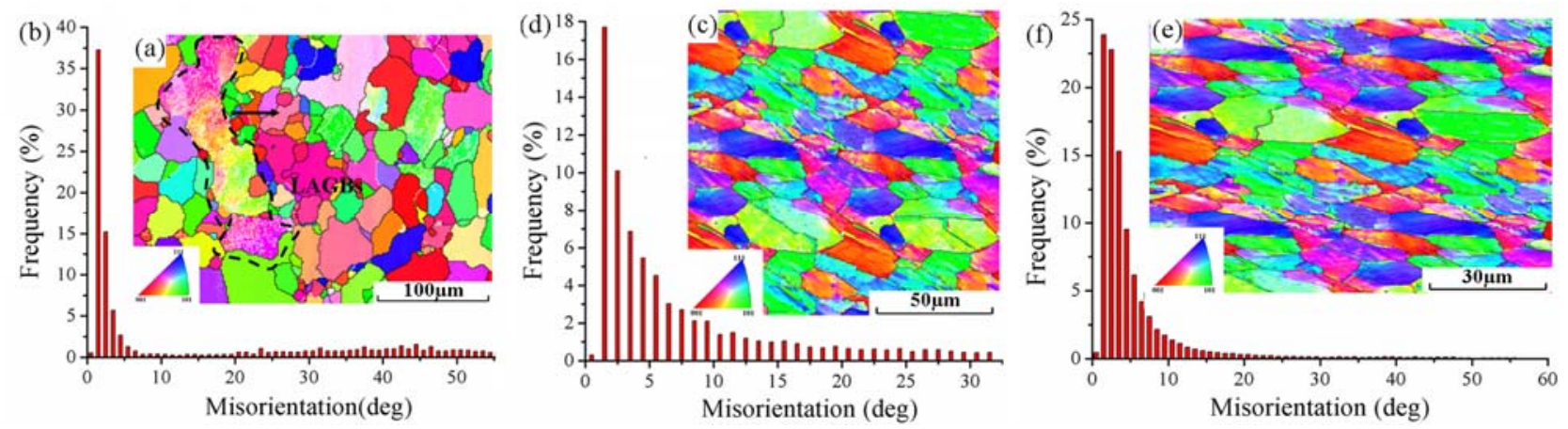

Figure 1 The undeformed pure Al: (a) inverse pole figure map, (b) grain boundary distribution; the 3 turns deformed pure Al: (c) inverse pole figure map, (d) grain boundary distribution; the 6 turns deformed pure Al: (c) inverse pole figure map, (d) grain boundary distribution

The average grain size of undeformed pure $\mathrm{Al}$ is about $50 \mu \mathrm{m}$, as shown in Fig. 1(a). In Fig. 1(a), the black grain boundaries refer to high angle grain boundaries (HAGBs). And most of the grains with HAGBs are presented. Meanwhile, large numbers of micro or nano scale substructures with low angle grain boundaries (LAGBs) gather inside some grains in Fig. 1(a) and Fig. 1(b). The grains are refined and elongated after 3 turns deformation observed from Fig. 1(c). The grain refinement and elongation are attributed to the accumulated plastic strain and introduction of shear stress. After 6 turns deformation, the grains are refined and elongated further observed from Fig. 1(e). The volume fraction of LAGBs in Fig. 1(f) increases in contrast to that in Fig. 1(b) and Fig. 1(d), which indicates that quantities of substructures with LAGBs distribute the grains. Yang et al. [8] indicated that the accumulated plastic strain was high due to the irreversibility of strain accumulation with the increase of deformation. After 9 turns deformation, the grain refinement become obvious and the volume percentage of LAGBs reaches steady state, as shown in Fig. 2(a), Fig. 2(b) and Fig. 2(c). The discontinuous HAGBs distribute non-uniformly on selected section, as shown in Fig. 2(a). The appearance of large numbers of dislocations and distortion of grain boundaries provide the driving force required for dynamic recovery and recrystallization [9]. The dynamic recrystallization (DRX) processes are divided into continuous dynamic recrystallization (CDRX) and discontinuous dynamic recrystallization (DDRX). The key distinguishing feature is that the obvious bulging of original grain boundaries could be observed during DDRX [10]. It can be seen that the bulging of original grain boundaries is observed from Fig. 2(a). The new grains occur when the nucleation strain is reached. And the initial microstructure is replaced completely at high strain. There is a gradual transformation during formation of subgrains. And the formed subgrains often appear in the vicinity of the grain boundaries, then subgrains evolve into grain nuclei with HAGBs [9]. It is inferred that the new formed grains are not obvious owing to limited accumulated plastic strain at room temperature, as illustrated Fig. 2(a). Also, the misorientation of subgrain boundary increases gradually and LAGBs are transformed into HAGBs. It can be seen from Fig. 2(a) that microstructure remains homogeneous during this process. 

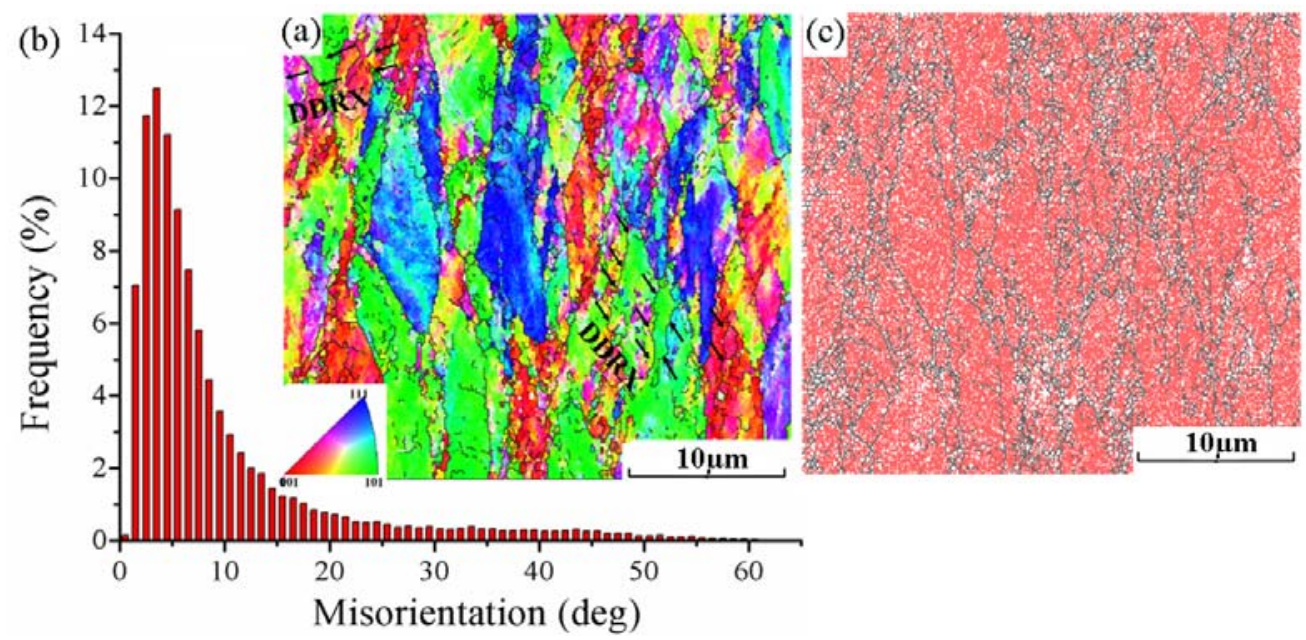

Figure 2 The 9 turns deformed pure Al: (a) inverse pole figure map, (b) and (c) grain boundary distribution.

\subsection{Texture Evolution}
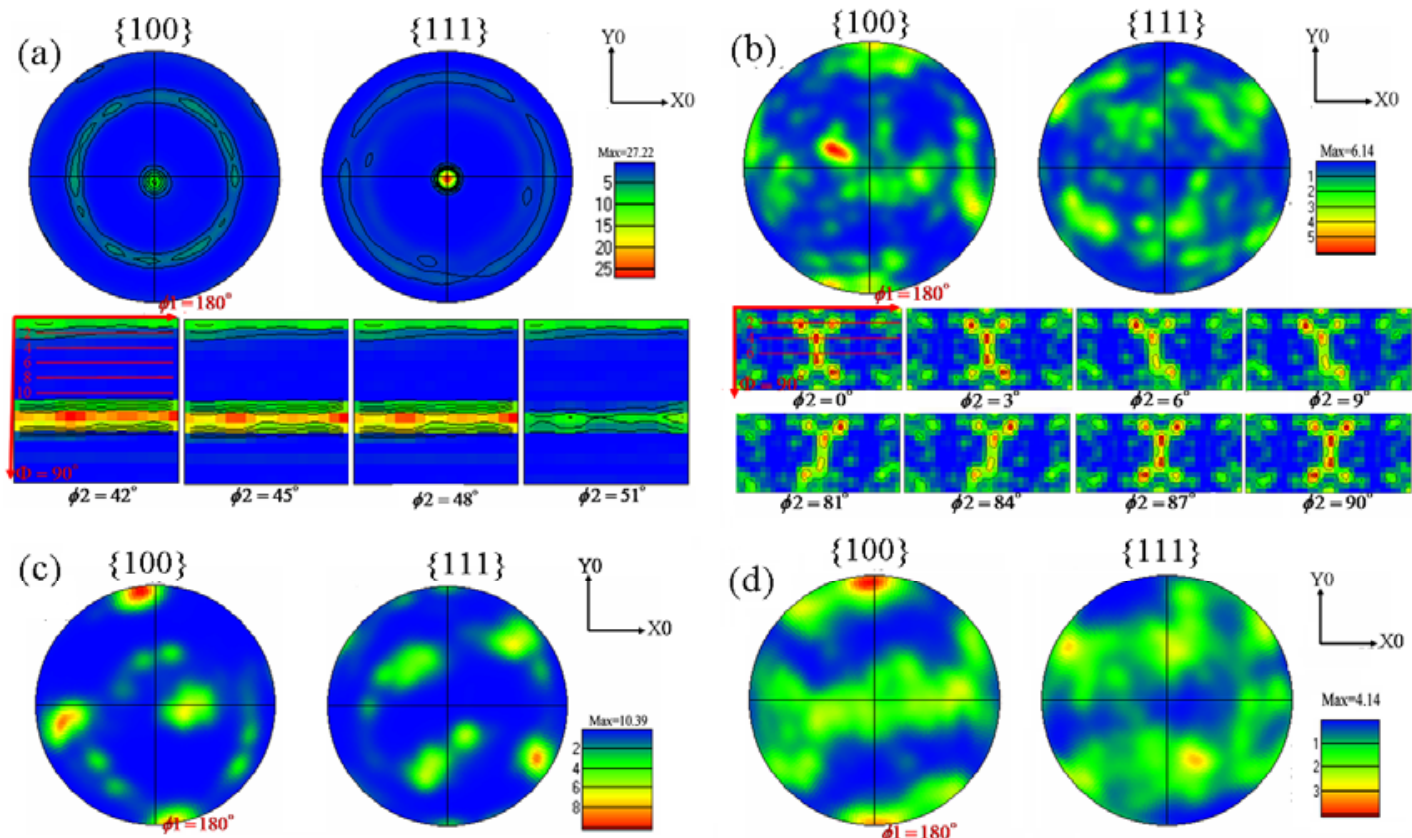

(d)

$\{100\}$
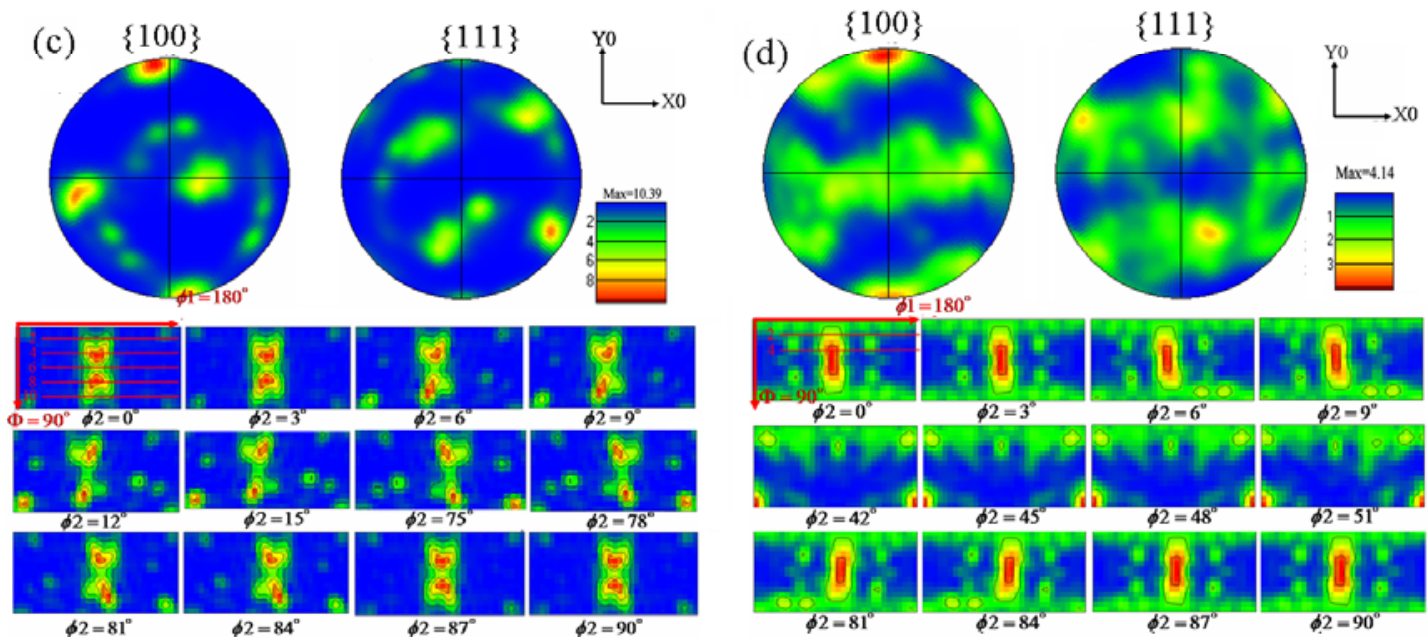

Figure 3 The pole figures and orientation distribution function of (a) undeformed pure Al, (b) 3 turns deformed pure Al, (c) 6 turns deformed pure Al and (d) 9 turns deformed pure Al.

The initial pole figures and orientation distribution function (ODF) of pure Al is shown in Fig. 3(a). It can be seen from Fig. 3(a) that the (111) pole point with maximum intensity locates in the center of the $\{111\}$ pole figure. Besides, (100) pole point is observed in the center of the $\{100\}$ pole figure. It is inferred that initial textures are mainly (111) and (001) fibers from the study of Alankar et al. [11]. And the Euler angles are mainly $\left(0-180^{\circ}, 54^{\circ}, 42-48^{\circ}\right)$. After 3 turns torsion deformation, the pole point with maximum intensity approaches to the centre of (100) pole figure, as shown in Fig. 3(b). Compared with that of Fig. 3(a), the intensity of (111) pole point decreases severely, but the intensity of (001) pole point increases obviously. The Euler angles are mainly $\left(90^{\circ}, 30^{\circ} / 60^{\circ}\right.$, 
$\left.0-9^{\circ} / 81-90^{\circ}\right)$ in Fig. 3(b), it is speculated that (001) fiber strengthens and (111) fiber weakens after 3 turns torsion deformation. Fig. 3(c) shows the pole figures and ODF of 6 turns deformed pure Al. $(100),(001),(0-10)$ and $(-100)$ pole points with different intensity are observed from $\{100\}$ pole figure. The intensity of (001) pole point remains almost unchanged, but the intensity of (100), (0-10) and $(-100)$ pole points increase, which indicates that $\{001\}$ fiber strengthens again compared with that in Fig. 3(b). At this time, the Euler angles are mainly $\left(90^{\circ}, 30^{\circ} / 60^{\circ}, 0-15^{\circ} / 75-90^{\circ}\right)$ in Fig. 3(c). With the increase of torsion deformation, typical (-100) and (100) pole points with certain intensity are observed from Fig. 3(d). The (001) and (0-10) pole points disappear compared with that in Fig. $3(\mathrm{c})$. Besides, the intensity of reserved $(-100)$ and (100) pole points decrease obviously and the position of pole point with maximum intensity rotates a slight angle in contrast to that in Fig. 3(c). And the Euler angles are mainly $\left(90^{\circ}, 30-60^{\circ}, 0-9^{\circ} / 42-51^{\circ} / 81-90^{\circ}\right)$, which can indicate that the $\{001\}$ fiber weakens with plastic deformation continuing. All this being said, the deformation texture of pure $\mathrm{Al}$ is mainly $\{001\}$ fiber during torsion. The position of $\{001\}$ fiber changes slightly, but the $\{001\}$ fiber strengthens first and then weakens with the increase of deformation.

\subsection{Dislocation Evolution}

The dislocation density increases and the boundaries of substructure are unclear for 3 turns deformed pure Al. Besides, lots of shear bands (SBs) were observed, for the original grains were elongated, as denoted by red stars in Fig. 4(a). The boundaries of substructure become clear and high density dislocations gather around the SBs with increasing deformation, as shown in Fig. 4(b). The dislocation density distributes non-uniformly on selected section and some dislocation tangle zones (DTZs) appear. With continuing plastic deformation, the SBs separated by dislocations evolve into large numbers of subgrain boundaries (SGBs), as shown in Fig. 4(c) and Fig. 4(d). The equiaxed cells or networks structures with high dislocation density appear, as denoted by A and B in Fig. 4(c). It can be seen from Fig. 4(c) that high density dislocation exists on the cell walls but low density dislocation distributes inside the cell. Also, the SGBs are surrounded by high dislocation density and the dislocation density in the subgrain structural elements is low, which suggests that these places undergo different levels of plastic deformation. In addition, fine grains zones with unclear grain boundaries are observed from Fig. 4(d). The grains are refined with the increase of accumulated plastic strain, but the accumulated plastic strain is not enough to form the nanostructured grains with clear GBs. The straight and narrow GBs, which are HAGBs formed by DRX combining with the former analysis. It is an equilibrium stress state under large strain caused by torsion deformation. In a word, the processes of grain refinement of coarse pure Al consists of formation of the SBs, SGBs, high-angle misorientation grain boundaries and equiaxed structures based on TEM observation and the previous researches [12-13].
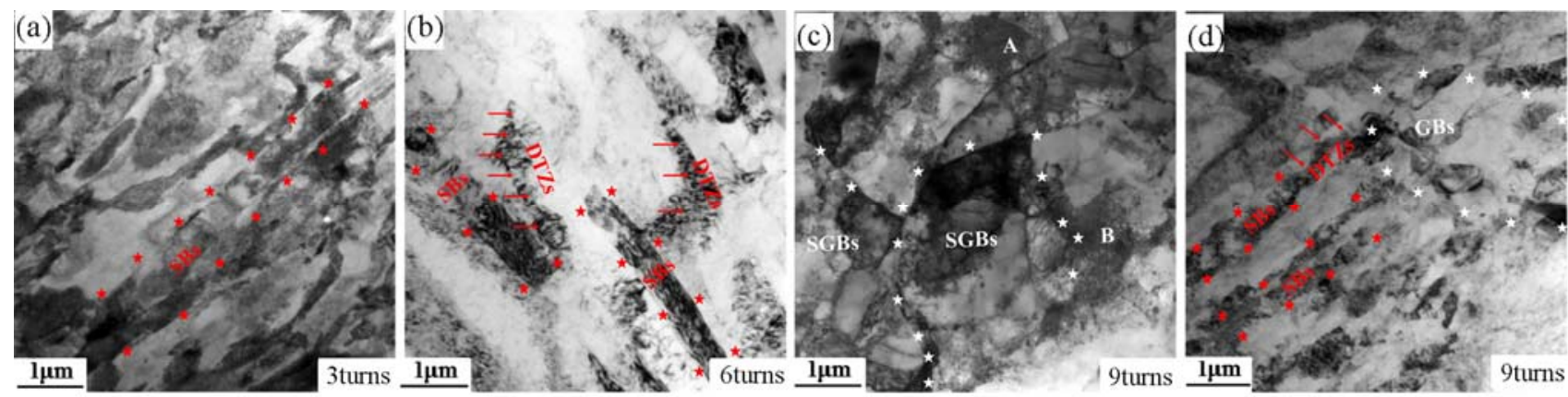

Figure 4 The TEM images of (a) 3 turns deformed pure Al, (b) 6 turns deformed pure Al, (c) and (d) 9 turns deformed pure Al.

\section{Conclusions}

The microstructure evolution and texture development of pure Al during torsion are investigated through experimental analysis. Some conclusions can be obtained as follows based on experimental results. 
(1) The grains of pure $\mathrm{Al}$ are refined gradually and the volume fraction of LAGBs increases with the increase of deformation during torsion. The dislocation induced refinement mechanism and recystallization (DDRX) induced refinement mechanism co-exist and lead to the grain refinement.

(3) The deformation texture of pure Al is mainly $\{001\}$ fiber. The position of $\{001\}$ fiber changes slightly, but the $\{001\}$ fiber strengthens first and then weakens with the increase of deformation during torsion.

(5) The TEM observation of pure $\mathrm{Al}$ indicates that the processes of grain refinement of pure $\mathrm{Al}$ consist of gradually forming of the SBs, SGBs, high-angle misorientation grain boundaries and equiaxed structures during torsion.

\section{References}

[1] Zhilyaev, A.P. and Langdon, T.G. (2008). Using high-pressure torsion for metal processing: fundamentals and applications. Progress in Materials Science, 53(6), 893-979.

[2] Zendehdel, H. and Hassani, A. (2012). Influence of twist extrusion process on microstructure and mechanical properties of 6063 aluminum alloy. Materials \& Design, 37, 13-18.

[3] Ungár, T., Tóth, L.S., Illy, J. and Kovács, I. (1986). Dislocation structure and work hardening in polycrystalline ofhc copper rods deformed by torsion and tension. Acta Metallurgica, 34(7), 1257-1267.

[4] Guo, N., Song, B., Guo, C.F., Xin, R.L. and Liu, Q. (2015). Improving tensile and compressive properties of magnesium alloy rods via a simple pre-torsion deformation. Materials \& Design, 83, 270-275.

[5] Chen, H., Li, F.G., Li, J.H., Ma, X.K., Li, J. and Wan, Q. (2016). Hardening and softening analysis of pure titanium based on the dislocation density during torsion deformation. Materials Science \& Engineering A, 671, 17-31.

[6] Suwas, S. and Ray, R.K. (2014). Crystallographic Texture of Materials. Springer London, Heidelberg New York Dordrecht, 1-8.

[7] Ivasishin, O.M., Kotrechko, S.O., Kucher, O.V., Ornatskty, A.I. and Shevchenko, S.V. (2009). Impact of a crystallographic texture of ti alloys on strength under tension and torsion. Metallofizika I Noveǐshie Tekhnologii, 31(5), 687-699.

[8] Yang, Z., Guo, Y.C., Li, J.P., He, F., Xia, F. and Liang, M.X. (2008). Plastic deformation and dynamic recrystallization behaviors of $\mathrm{mg}-5 \mathrm{gd}-4 \mathrm{y}-0.5 \mathrm{zn}-0.5 \mathrm{zr}$ alloy. Materials Science \& Engineering A, 485(1-2), 487-491.

[9] Sakai, T., Belyakov, A., Kaibyshev, R., Miura, H. and Jonas, J.J. (2014). Dynamic and post-dynamic recrystallization under hot, cold and severe plastic deformation conditions. Progress in Materials Science, 60(1), 130-207.

[10] Xin, R.L., Zheng, X., Liu, Z., Liu, D.J., Qiu, R.S., Li, Z.Y. and Liu, Q. (2016). Microstructure and texture evolution of an $\mathrm{mg}-\mathrm{gd}-\mathrm{y}-\mathrm{nd}-\mathrm{zr}$ alloy during friction stir processing. Journal of Alloys \& Compounds, 659, 51-59.

[11] Alankar, A., Mastorakos, I.N. and Field, D.P. (2009). A dislocation-density-based 3d crystal plasticity model for pure aluminum. Acta Materialia, 57(19), 5936-5946.

[12] Chen, H., Li, F.G., Li, J.H., Zhao, Z., Zhou, S.S. and Wan, Q. (2016). Experimental study on pure titanium during the positive-torsion and positive-negative-torsion. Materials Science \& Engineering A, 674, 552-568.

[13] Khamsuk, S., Park, N., Adachi, H., Terada, D. and Tsuji, N. (2012). Evolution of ultrafine microstructures in commercial purity aluminum heavily deformed by torsion. Journal of Materials Science, 47(22), 7841-7847. 